\title{
Usability Evaluation of a One-Handed Touch-based OR-Table Control
}

Philipp Krumholz, Chair of Medical Engineering at Helmholtz-Institute for Biomedical Engineering, RWTH Aachen University, 52074 Aachen, Germany, krumholz@hia.rwth-aachen.de Armin Janß, Chair of Medical Engineering at Helmholtz-Institute for Biomedical Engineering, RWTH Aachen University, 52074 Aachen, Germany, janss@ @urgitaix.com

Klaus Radermacher, Chair of Medical Engineering at Helmholtz-Institute for Biomedical Engineering, RWTH Aachen University, 52074 Aachen, Germany, radermacher@hia.rwth-aachen.de

Due to the increasing number and the complexity of devices in the operation room (OR), which are caused by the diversity and variety of proprietary interface designs, the probability for use errors increases. In order to ensure a safe intraoperative workflow in the OR, integrated OR systems with central work stations have been developed. For this, there also exist concepts for handhelds, which offer touch-based graphical user interfaces (GUI). However, those come along with various limitations, e.g. possibility for one-handed interaction, which is influenced i.a. by the size, the design and the working task. Within our investigations, requirements for an enhanced concept have been gathered by taking into account corresponding standards regarding ergonomics, risk-management and usability-engineering. According to DIN EN 60601-2-46, machinery directive 2006/42 EG and guideline VDI/VDE 3850, the stipulated user interface has been designed in order to preselect functions on the touchscreen and their subsequent activation by physical buttons. Furthermore, the accessibility of areas of interest has been considered on the touchscreen, to avoid the re-gripping of the handheld. As a first step, the ORtable control for a tablet has been realized with Microsoft Blend, using a 22' touch-monitor. The usability study has been conducted in accordance with DIN EN ISO 9241-11 and IEC 62366. A group of ten surgeons and OR nurses underwent multiple tasks in the framework of a usability test, comparing the developed concept with a common keypad handheld. The study showed comparable results concerning the usability criteria effectiveness and efficiency. However, the concept clearly outperforms the handheld regarding user satisfaction, in particular due to the higher learnability and the easy transfer of daily-used GUI skills. The current approach motivates to develop a functional model of a touch-based handheld for OR-tables as well as with further integrated OR devices and to con-duct additional usability studies. 


\section{Postural workloads on paramedics during patient transport}

Mark Verjans, Chair of Medical Engineering at Helmholtz-Institute for Biomedical Engineering of the RWTH Aachen University, Aachen, Germany, verjans @ hia.rwth-aachen.de Andrea Schütt, Chair of Medical Engineering at Helmholtz-Institute for Biomedical Engineering of the RWTH Aachen University, Aachen, Germany, andrea.schuett@alumni.fh-aachen.de

Philipp Schleer, Chair of Medical Engineering at Helmholtz-Institute for Biomedical Engineering of the RWTH Aachen University, Aachen, Germany, schleer@hia.rwth-aachen.de Detlef Struck, Rettungsdienst Kreis Düren AöR (Emergency medical services of Düren district), Kreuzau, Germany, d.struck@kreis-dueren.de

Klaus Radermacher: Chair of Medical Engineering at Helmholtz-Institute for Biomedical Engineering of the RWTH Aachen University, Aachen, Germany, radermacher@hia.rwth-aachen.de

Paramedics perform physically demanding tasks during patient transport in daily routine and therefore suffer more often from musculoskeletal ailments, mainly low back pain, than any other profession. We hypothesise, that current transport aids do not offer sufficient support when it comes to obstacles and stairs during patient transport. Therefore we con-ducted an Ovako Working Posture Analysing System (OWAS) field study to capture postural workloads during patient transport and connected the results to a survey among paramedics about occurring obstacles. The results of the OWAS analysis showed strenuous working conditions during barrier-free transport with classical transport aids, like stretcher and stretcher chair, but enormous postural workloads when barriers occurred. Our survey revealed, that stairs occurred in $38 \%$, and at least one barrier, like narrow passages, curbs, etc., in $48.1 \%$ of all deployments $(\mathrm{n}=405)$, we can quantitatively link postural workloads with occurring obstacles. In conclusion, there is a high demand for ergonomic improvements of current transport aids and a high potential of active assist devices to reduce harmful loads on paramedics. 


\section{Assessment of Natural User Interactions for Robot Assisted Interventions}

Johann Berger, Innovation Center Computer Assisted Surgery, Leipzig, Germany, johann.berger@medizin.unileipzig.de

Michael Unger, Innovation Center Computer Assisted Surgery, Leipzig, Germany, michael.unger@medizin.unileipzig.de

Lisa Landgraf, Innovation Center Computer Assisted Surgery, Leipzig, Germany, lisa.landgraf@medizin.uni-leipzig.de Richard Bieck, Innovation Center Computer Assisted Surgery, Leipzig, Germany, richard.bieck@medizin.unileipzig.de

Thomas Neumuth, Innovation Center Computer Assisted Surgery, Leipzig, Germany, thomas.neumuth@medizin.unileipzig.de

Andreas Melzer, Innovation Center Computer Assisted Surgery, Leipzig, Germany, andreas.melzer@medizin.unileipzig.de

Robotic systems provide the means to support the surgeon in tiring and repetitive tasks with very high precision over a long time span. However, due to complicated interfaces the user acceptance remains low. Collaborative robotics propose effective concepts to overcome this problem. Flexible robotic arms provide the versatility to be used in a wide range of various treatments and concepts for device interaction need to be investigated. Therefore, the aim of this work is to analyze the recognition accuracy of simple force-feedback based touch commands on a robotic arm.

For this study a robotic platform comprising a KUKA LBR iiwa 7 R800 robotic arm (Kuka AG, Augsburg, Germany), the standard Sunrise Cabinet console and an additional CPU was built. The robot is mounted on a compact rack and a Clarius L7 (Clarius, Canada) mobile ultrasound probe is mounted to the flange as an exemplary tool. A forcerecognition application to read touch gestures at the endeffector was implemented in $\mathrm{C}++$ using the Robot Operating System (ROS).

Eigth particiants evaluated the accuracy of the touch gesture recognition. Four different touch gestures were examined: left push, right push, left double tap and right double tap. The participants had to perform each interaction 25 times in random order. To gather information on usability and acceptance, a simple questionnaire had to be answered. The evaluation resulted in a $89.8 \%$ accuracy. Seven participants ranked the intuitiveness as high or very high. All found the idea to be good or very good.

The gesture recognition application presented in this work enables users to naturally interact with robotic systems. Shown accuracy values are promising and can be increased further by applying more sophisticated methods to filter error prone signals. A use case specific demonstrator will be developed to evaluate workloads and time savings in a preclinical study. 


\title{
Analysis and Simulation of the Illumination Optics of Rigid Medical Endoscopes
}

\author{
Alexander Gaertner, Institute of Technical Medicine, Furtwangen University, Villingen-Schwenningen, \\ Germany, A.Gaertner@hs-furtwangen.de \\ Paola Belloni, Institute of Technical Medicine, Furtwangen University, Villingen-Schwenningen, Germany, \\ Paola.Bel-loni@hs-furtwangen.de
}

Our work focuses on enhancing the illumination optics of a rigid medical endoscope using optomechanical simulation. We aim on improving the efficiency of the illumination optics to provide more light in the surgeon's field of view (FOV) and therefore better image quality. We first conducted an extensive market analysis and measured several rigid endo-scopes and their external light sources in the lighting technology laboratory. Surprisingly, all showed a degree of efficiency below $20 \%$. The low efficiency is due to absorption, scattering and reflection within the endoscope's illumination optics. These optical effects result not only in decreased light output in the FOV but also in undesirable conversion from luminous to thermal energy.

Our optimization approach is based first on photometric and colorimetric analysis carried out with an integrating sphere and a photogoniometer. The results obtained are implemented in an 3D-optomechanical simulation model developed with the raytracing software LightTools (Synopsis(C). In the simulation model both the geometrical components as well as the critical interfaces are examined considering light coupling at boundary surfaces and light transfer within the illumination optics. The optimization is executed in LightTools taking into account the spectral properties and the angular distribution of the light emitted by the fiberoptic as well as additional interfering factors like gluing, dispersive absorption and total reflection at critical angles.

Thus, a trade-off of the simulation results is performed by weighting up between the best possible optical solution and their feasibility with conventional production technology. Finally, a prototype will be realized in collaboration with a partner company by integrating the optimized illumination optics into a rigid medical endoscope. The optimized prototype will be of course validated in our lighting technology laboratory. 


\section{Integrating a Usability Engineering Process into a Consisting Risk Management}

Michael Scholtes, University of Applied Sciences - Faculty of Health Sciences, Wiesenstraße 14, 35390 Gießen, Germany, e-mail: Michael.Scholtes@ges.thm.de

Stephanie Buedenbender, University of Applied Sciences - Faculty of Health Sciences, Wiesenstraße 14, 35390 Gießen, Germany, e-mail: Stephanie.Buedenbender@ges.thm.de

Annemarie Behrend, University of Applied Sciences - Faculty of Health Sciences, Wiesenstraße 14, 35390 Gießen, Germany, e-mail: Annemarie.Behrend@ges.thm.de

Keywan Sohrabi, University of Applied Sciences - Faculty of Health Sciences, Wiesenstraße 14, 35390 Gießen, Germany, e-mail: Keywan.Sohrabi@ges.thm.de

Volker Gross, University of Applied Sciences - Faculty of Health Sciences, Wiesenstraße 14, 35390 Gießen, Germany, e-mail: Volker.Gross@ges.thm.de

The complexity of medical devices and its user interactions increases. A growing number of incident reports are assumed to be associated primarily with user errors. This development is tackled through current modifications in standards, such as ISO 13485:2016 and legislations, such as the Medical Device Regulation. Both intensify the focus on use errors sig-nificantly. The aim of this paper was the development of a process orientated approach integrating usability engineering into a consisting risk management based on a classic V-model. An appropriate procedure was worked out. For each development step, risk and usability activities were cumulated. Thus, the present paper might help medical device manu-facturers to reflect their risk management and usability management processes to find synergies. Prospectively, a step-by-step guide for the integration of risk management and usability engineering based on this approach should be devel-oped. 


\section{Steps toward digitization of medical devices}

Andreas Zimolong, Synagon GmbH, Aachen, Germany, Andreas.Zimolong@Synagon.de

Sandra Fiehe, Synagon GmbH, Aachen, Germany, Sandra.Fiehe@ Synagon.de

Peter Knipp, qcmed GmbH, Niederkassel, Germany, P.Knipp@qcmed.de

Digitization of business processes and business models has for sure reached the health care system, but many medical device manufacturers still cling to the old times with systems residing on their own data island and not communicating anything. Nevertheless medical devices now have to exchange data and to be open for remote management while ensuring security of the data network.

In the BMBF project OR.NET a secure dynamic networking of components in operating room and clinic has been devel-oped. The succeeding project MoVE focuses on the approval and certification process of these medical devices connected in dynamic data networks. It aims to provide a test platform to prove conformity to standards (especially IEEE 11073-SDC), thus enabling manufacturers and operators to verify their devices and interfaces. Documented evidence of regula-tory compliance is necessary in order to enable networking and to release medical devices from their data island.

For conformity assessment of medical devices to be integrated in dynamic networks a defined reference is needed. Within the MoVE project this reference is devoloped and implemented in form of a test environment and associated test scenarios. Requirements of the test platform are documented in requirements specifications of different degree of detail and per-spectives. Test scenarios are defined based on the standard as well as from a risk assessment perspective. The whole test environment needs to be validated against the requirements and needs to ensure valid and reproducible test results.

When talking about user requirements, the different use scenarios have to be kept in mind. Next to an accredited lab which certifies conformity to standards, the test environment is to be used by medical device manufacturers during device de-velopment as well as by medical device operators to verify safe \& secure operation of networked devices. These different use cases however result in different perspectives to be integrated in the User Requirements Specification (URS). 


\section{Innovative Ventilation Technology for Operating Rooms}

Sabine Gruber and Sebastian Buhl (Ostbayerische Technische Hochschule Amberg-Weiden, Germany); Clemens Bulitta (Ostbayerische Technische Hochschule (OTH), Germany)

s.gruber@oth-aw.de

The purpose of this work was to evaluate the decontamination potential of the Potok system both in an experimental setting in a research Operating Room (OR) with standalone Air Decontamination Units (Potok 150-M-01) and in a clin-ical setting in a real operating theater in Moscow. Our experiments showed an impact of the Potok units on the bacterial contamination of the room air according to the Swedish SIS-TS 39:2015

standard. For the initial measurements in our research OR in Weiden this could be shown by a decrease of the bacterial burden at all three different measurement points (OR table, Instrument board, Periphery). For the measurement directly on the OR table the activity of the units led to a decrease of the bacterial air contamination from $5 \mathrm{cfu} / \mathrm{m}^{3}$ to $3 \mathrm{cfu} / \mathrm{m}^{3}$ in average. For the Instrument table and the Periphery of the room this reduction was from $5 \mathrm{cfu} / \mathrm{m}^{3}$ and $12 \mathrm{cfu} / \mathrm{m}^{3}$ down to $4 \mathrm{cfu} / \mathrm{m}^{3}$ and $5 \mathrm{cfu} / \mathrm{m}^{3}$ respectively.

Also the subsequently done measurements in the Moscow hospital verified this decontaminating effectivity of the Potok system. In this case the initial background contamination of the operating theater was higher than in the research OR in Germany. This bacterial burden could be effectively decreased by the use of the installed Potok based ventilation sys-tem. For the three different measurement locations our results showed a decrease of more than $87 \%$. The initial bacterial burden of $37 \mathrm{cfu} / \mathrm{m}^{3}$ on the OR table and $39 \mathrm{cfu} / \mathrm{m}^{3}$ on the instrument board and the periphery of the room had been re-duced to $\leq 5 \mathrm{cfu} / \mathrm{m}^{3}$ in average for every measurement point.

Based on our findings with the Potok system it should be discussed whether the technology has to be considered as a viable alternative to other currently used ventilation systems and whether it represents another potential solution for in-fection control of airborne microbiological burden of operating theaters. 